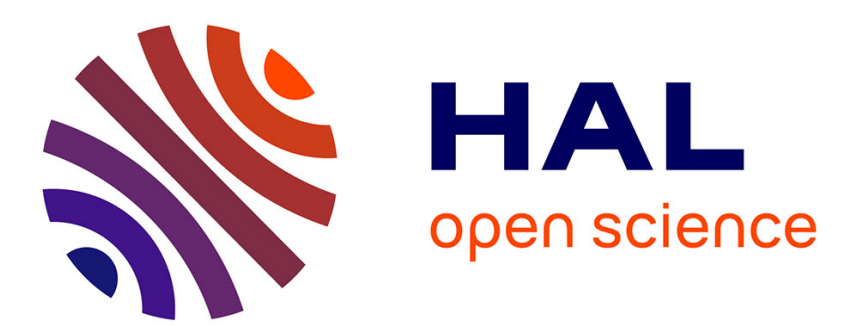

\title{
3D BEM Formulations for Eddy Current Problems with Multiply Connected Domains and Circuit Coupling
}

\author{
Quang-Anh Phan, Gérard Meunier, Jean-Michel Guichon, Bertrand
}

Bannwarth, Olivier Chadebec

\section{- To cite this version:}

Quang-Anh Phan, Gérard Meunier, Jean-Michel Guichon, Bertrand Bannwarth, Olivier Chadebec. 3D BEM Formulations for Eddy Current Problems with Multiply Connected Domains and Circuit Coupling. IEEE Transactions on Magnetics, 2022, 58 (4), 10.1109/TMAG.2019.2954323 . hal-03013841

\section{HAL Id: hal-03013841 \\ https://hal.science/hal-03013841}

Submitted on 19 Nov 2020

HAL is a multi-disciplinary open access archive for the deposit and dissemination of scientific research documents, whether they are published or not. The documents may come from teaching and research institutions in France or abroad, or from public or private research centers.
L'archive ouverte pluridisciplinaire HAL, est destinée au dépôt et à la diffusion de documents scientifiques de niveau recherche, publiés ou non, émanant des établissements d'enseignement et de recherche français ou étrangers, des laboratoires publics ou privés. 


\title{
3D BEM Formulations for Eddy Current Problems with Multiply Connected Domains and Circuit Coupling
}

\author{
Quang-Anh Phan ${ }^{1}$, Olivier Chadebec ${ }^{1}$, Gerard Meunier ${ }^{1}$, Jean-Michel Guichon ${ }^{1}$, Bertrand Bannwarth ${ }^{1}$ \\ ${ }^{1}$ University Grenoble Alpes, CNRS, Grenoble INP, G2Elab, 38000 Grenoble, France
}

\begin{abstract}
Quasi-static linear problems can be solved efficiently with Boundary Element Method (BEM). This method is based on surface integral equations dealing with equivalent magnetic and electric surface current densities. Many works have shown the potentiality of BEM especially for the modeling of non-destructive testing devices. In this paper, after selecting formulations enabling the modeling of multiply-connected regions, an original coupling is proposed in order to take into account external electric circuit in the problem.
\end{abstract}

Index Terms-Boundary Element Method, eddy currents, quasi-static, circuit coupling.

$\mathbf{T}$ HIS study is motivated by the need of efficient quasistatic electromagnetic formulations associated to nonsimply connected domains and which can be coupled with circuit equations. For this kind of problems, methods based on coupling between BEM and Finite Element Method (FEM) have already shown very good results. However, the accuracy of these methods is strongly influenced by the volume mesh of the conductive region which has to be adapted to the skindepth in the FEM context. The pure BEM method does not suffer from this disadvantage because it is only based on surface discretizations and is more insensitive to frequency increase keeping the same mesh.

Many BEM formulations have already been proposed to solve Eddy Current (EC) problems and two options are possible. The first one consists in solving the full Maxwell equations including capacitive effects and to use an HelmholtzHodge decomposition to remove low-frequency numerical noise like proposed in [1]. The second option is to solve directly Maxwell equations under the quasi-static assumption (i.e the EC problem) as proposed in [3],[4] or [5]. In this work, the preference is given to the last approach.

In classical EC problems (like eddy current testing problems for instance), the active region can be multiply connected, meaning that it can have through-holes. It is fundamental to develop formulations which can solve accurately such a problem. An another key point is the ability of the formulation to be coupled with external electric circuits which create the external inductor field. This is what we aim to do in this paper. After selecting efficient BEM formulations for EC problems, an original circuit coupling is proposed. All the formulations are validated on numerous examples by a comparison with FEM.

\section{SURFACE INTEGRAL FORMULATIONS}

\section{A. Integral Equations}

Let us consider a linear, isotropic and homogeneous conducting material region $\Omega$ characterized by the conductivity $\sigma_{1}$ and the permeability $\mu_{1}$ and embedded in the free space $\Omega_{0}$. $\Gamma$ is the border of the region $\Omega$ with the outward unit normal $\mathbf{n}$. Surface integral equations of $\mathbf{E}$ and $\mathbf{H}$ at an arbitrary target point $\mathrm{P}$ in $\Omega_{0}$ are :

$$
\begin{aligned}
h \mathbf{H}= & \mathbf{H}^{s}+\int_{\Gamma}\left(\left(\mathbf{n} \cdot \mathbf{H}_{0}\right) \nabla G_{0}+\mathbf{J}_{s} \times \nabla G_{0}\right) d \Gamma \\
h \mathbf{E}= & \mathbf{E}^{s}+\int_{\Gamma}\left(\left(\mathbf{n} \cdot \mathbf{E}_{0}\right) \nabla G_{0}-\mathbf{M}_{s} \times \nabla G_{0}\right) d \Gamma \\
& -j \omega \mu_{0} \int_{\Gamma} \mathbf{J}_{s} G_{0} d \Gamma
\end{aligned}
$$

and for a target point in $\Omega$ are :

$$
\begin{aligned}
h \mathbf{H}= & -\int_{\Gamma}\left(\frac{\mu_{0}}{\mu_{1}}\left(\mathbf{n} \cdot \mathbf{H}_{0}\right) \nabla G_{1}-\sigma_{1} \mathbf{M}_{s} G_{1}\right) d \Gamma \\
& -\int_{\Gamma} \mathbf{J}_{s} \times \nabla G_{1} d \Gamma \\
h \mathbf{E}= & \int_{\Gamma}\left(\mathbf{M}_{s} \times \nabla G_{1}+j \omega \mu_{1} \mathbf{J}_{s} G_{1}\right) d \Gamma
\end{aligned}
$$

where $h=0.5$ if $\mathrm{P}$ is a regular point of the border $\Gamma, h=1$ if $\mathrm{P}$ belongs to $\Omega$ or $\Omega_{0}$. The Green kernels expressions are $G_{0}=\frac{1}{r}$ and $G_{1}=\frac{1}{4 \pi r} e^{-(1+j) k r}$ with $k=\sqrt{\frac{\omega \mu_{1} \sigma_{1}}{2}}, \mathbf{H}_{0}$ and $\mathbf{E}_{0}$ are the magnetic and electric fields in the air region respectively, $\mathbf{H}^{s}$ and $\mathbf{E}^{s}$ denote the electric and magnetic vectors of the incident field respectively, expressed by the current density of source $\mathbf{j}^{s}$ as:

$$
\begin{aligned}
& \mathbf{H}^{s}=\int_{\Omega^{s}} \mathbf{j}^{s} \times \nabla G_{0} d \Omega \\
& \mathbf{E}^{s}=-j \omega \int_{\Omega^{s}} \mathbf{j}^{s} G_{0} d \Omega
\end{aligned}
$$

where $\Omega^{s}$ is source current region, $\mathbf{J}_{s}$ and $\mathbf{M}_{s}$ are equivalent magnetic and electrical surface currents such as :

$$
\begin{aligned}
\mathbf{J}_{s} & =\mathbf{n} \times \mathbf{H} \\
\mathbf{M}_{s} & =-\mathbf{n} \times \mathbf{E}
\end{aligned}
$$

For the sake of clarity, we use some definitions as follows:

$$
\begin{aligned}
& \mathcal{A}^{\ell}(\mathbf{U})=p \cdot v \cdot \int_{\Gamma} \mathbf{U} G_{\ell} d \Gamma \quad \mathcal{B}^{\ell}(\mathbf{U})=p \cdot v \cdot \int_{\Gamma} \mathbf{U} \times \nabla G_{\ell} d \Gamma \\
& \mathcal{C}^{\ell}(u)=-p \cdot v \cdot \int_{\Gamma} u \nabla G_{\ell} d \Gamma
\end{aligned}
$$




$$
\begin{aligned}
\mathcal{A}_{\times}^{\ell}(\mathbf{U}) & =\mathcal{A}^{\ell}(\mathbf{U}) \times \mathbf{n}, & \mathcal{A}_{n}^{\ell}(\mathbf{U}) & =\mathcal{A}^{\ell}(\mathbf{U}) \cdot \mathbf{n}, \\
\mathcal{B}_{\times}^{\ell}(\mathbf{U}) & =\mathcal{B}^{\ell}(\mathbf{U}) \times \mathbf{n}, & \mathcal{B}_{n}^{\ell}(\mathbf{U}) & =\mathcal{B}^{\ell}(\mathbf{U}) \cdot \mathbf{n}, \\
\mathcal{C}_{\times}^{\ell}(u) & =\mathcal{C}^{\ell}(u) \times \mathbf{n}, & \mathcal{C}_{n}^{\ell}(u) & =\mathcal{C}^{\ell}(u) \cdot \mathbf{n},
\end{aligned}
$$

where $p . v$. denote Cauchy principal value, index $\ell \in\{0,1\}$.

From the four equations (1)-(4) and by considering their normal and tangential projections on $\Gamma$, we get eight integral boundary equations.

\section{On the outer side:}

$$
\begin{aligned}
\mathbf{E}_{\times}^{s} & =\frac{1}{2} \mathbf{M}_{s}+i \omega \mu_{0} \mathcal{A}_{\times}^{0}\left(\mathbf{J}_{s}\right)+\mathcal{B}_{\times}^{0}\left(\mathbf{M}_{s}\right)+\mathcal{C}_{\times}^{0}\left(\mathbf{E}_{0 n}\right) \\
\mathbf{H}_{\times}^{s} & =-\frac{1}{2} \mathbf{J}_{s}-\mathcal{B}_{\times}^{0}\left(\mathbf{J}_{s}\right)+\mathcal{C}_{\times}^{0}\left(\mathbf{H}_{0 n}\right) \\
\mathbf{E}_{n}^{s} & =\frac{1}{2} \mathbf{E}_{0 n}+j \omega \mu_{0} \mathcal{A}_{n}^{0}\left(\mathbf{J}_{s}\right)+\mathcal{B}_{n}^{0}\left(\mathbf{M}_{s}\right)+\mathcal{C}_{n}^{0}\left(\mathbf{E}_{0 n}\right) \\
\mathbf{H}_{n}^{s} & =\frac{1}{2} \mathbf{H}_{0 n}-\mathcal{B}_{n}^{0}\left(\mathbf{J}_{s}\right)+\mathcal{C}_{n}^{0}\left(\mathbf{H}_{0 n}\right)
\end{aligned}
$$

On the inner side:

$$
\begin{aligned}
0 & =-\frac{1}{2} \mathbf{M}_{s}+j \omega \mu_{1} \mathcal{A}_{\times}^{1}\left(\mathbf{J}_{s}\right)+\mathcal{B}_{\times}^{1}\left(\mathbf{M}_{s}\right) \\
0 & =\frac{1}{2} \mathbf{J}_{s}-\mathcal{B}_{\times}^{1}\left(\mathbf{J}_{s}\right)+\sigma_{1} \mathcal{A}_{\times}^{1}\left(\mathbf{M}_{s}\right)+\mathcal{C}_{\times}^{1}\left(\mathbf{H}_{1 n}\right) \\
0 & =j \omega \mu_{1} \mathcal{A}_{n}^{1}\left(\mathbf{J}_{s}\right)+\mathcal{B}_{n}^{1}\left(\mathbf{M}_{s}\right) \\
0 & =-\frac{1}{2} \mathbf{H}_{1 n}-\mathcal{B}_{n}^{1}\left(\mathbf{J}_{s}\right)+\sigma_{1} \mathcal{A}_{n}^{1}\left(\mathbf{M}_{s}\right)+\mathcal{C}_{n}^{1}\left(\mathbf{H}_{1 n}\right)
\end{aligned}
$$

Combining these integral equations by multiplying (6) (13) respectively by the weighting factors $\alpha_{\ell}, \beta_{\ell}, a_{\ell}, b_{\ell}$ and summing contributions of $\Omega_{0}$ and $\Omega$, we obtain the system of six formulations given by Tab. [1]

The obtained formulations called from EC1 to EC6. EC1 and EC2 have already been presented in [3]. EC4, EC5 with a combination such as the PMCWHT formulation was presented in [1], [5]. EC5 was proposed in [4], the idea of combination comes from formulation of Muller [7]. The EC6 formulation has been added to complete the set of combinations.

\section{B. Choice of BEM formulation}

Formulations in Tab. I are now examined in the context of a low frequency eddy current problem. A conductive ring $\left(r_{\text {int }}=4 \mathrm{~mm}, r_{\text {ext }}=10 \mathrm{~mm}, h=2 \mathrm{~mm}\right)$ is considered. An excitation coil is placed $1 \mathrm{~mm}$ above $\left(r_{\text {int }}=6.75 \mathrm{~mm}, r_{\text {ext }}=\right.$ $7.25 \mathrm{~mm}, h=0.5 \mathrm{~mm}, I=10 \mathrm{~A}, 1$ turn $)$. The surface of the ring is discretized by 1792 quadrangle elements. The current density $\mathbf{M}_{s}, \mathbf{J}_{s}$ are interpolated by the facet shape function (2-form Whitney elements) and the 0-form Whitney elements for $\mathbf{E}_{0 n}$.

$$
\mathbf{M}_{s}=\sum_{i=1}^{n_{M}} \mathbf{w}_{i} M_{s i}, \quad \mathbf{J}_{s}=\sum_{i=1}^{n_{J}} \mathbf{w}_{i} J_{s i}, \quad \mathbf{E}_{0 n}=\sum_{i=1}^{n_{E}} \alpha_{i} E_{0 n i} .
$$

where $n_{M}, n_{J}, n_{E}$ are numbers of degree of freedom corresponding to $\mathbf{M}_{s}, \mathbf{J}_{s}$ and $\mathbf{E}_{0 n}$.

Using the Galerkin approach, we perform calculations with frequencies ranging from $10 \mathrm{~Hz}$ to $100 \mathrm{kHz}$ for each formulation. The reference result is computed by axisymmetric finite element method. Figure 1 shows the relative loss in three cases respectively: $\sigma=5.5 E 7, \mu_{r}=1$ (case 1), $\sigma=5.5 E 6, \mu_{r}=10$ (case 2), $\sigma=5.5 E 5, \mu_{r}=100$ (case 3). The skin depth varies from $21.46 \mathrm{~mm}$ to $0.21 \mathrm{~mm}$ in these cases. The tests carried out show that the EC1 formulation

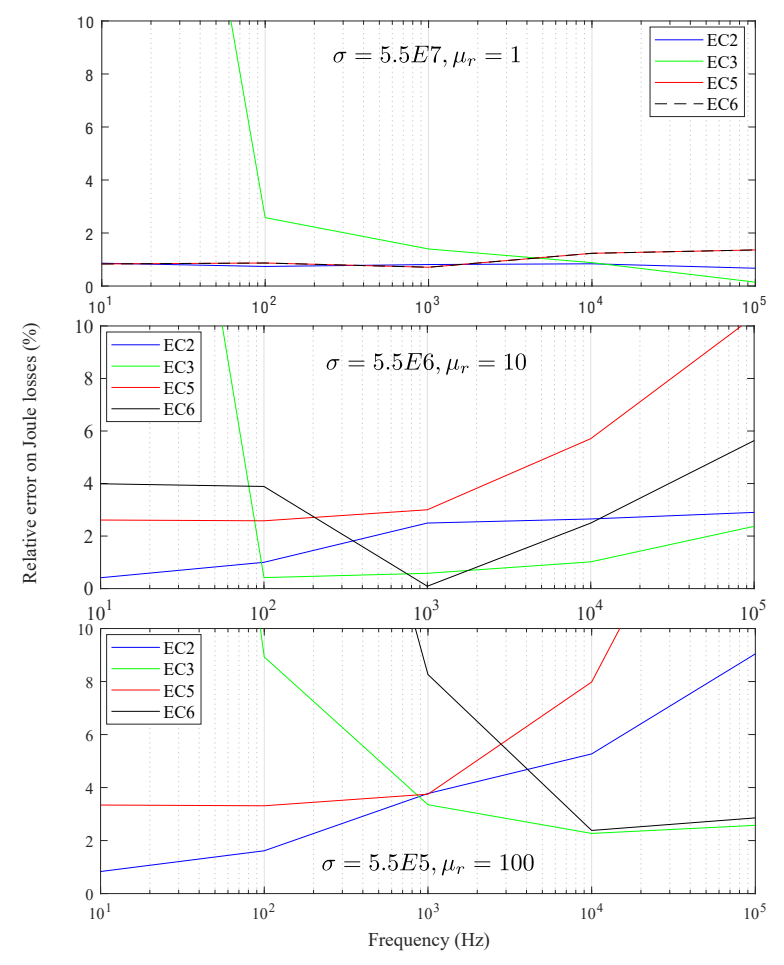

Fig. 1. Error on Joule losses. Comparison of different BEM formulations with axisymetric FEM problem.

does not work in this case multiply-connected regions, the current distribution obtained being wrong. That's why we don't show results of EC1 formulations. For the circuit coupling problem, it is necessary to have the magnetic potential A outside the conductor (see next section) which depends on $\mathbf{E}_{0 n}$. In EC4, this quantity is not computed. That's why we didn't test this formulation. The number of candidate formulations for circuit coupling is then reduced to EC2, EC3, EC5 and EC6, which are compatible with multiply connected problems and enable the computation of the magnetic vector potential outside. In the case of non-magnetic conductivity, the results obtained with EC2, EC5 and EC6 lead to a better accuracy than EC3 up to a few $\mathrm{kHz}$. However, with a higher frequency, EC3 becomes more accurate. In the case of conductive and magnetic material, when relative permeability is high at 100 , all three formulations give poor results, especially for EC3, EC6 at a frequency below $10 \mathrm{kHz}$ and for EC2, EC5 at a frequency above $1 \mathrm{kHz}$. With a lower magnetic permeability $\mu_{r}=10$, EC2 gives better results than the others in the frequency range considered.Let us notice that if an iterative solver like GMRES is used, EC6 presents the best convergence rate.

\section{Circuit COUpling}

\section{A. Electrical circuit equations}

In this part, the construction of the equation dealing with imposed voltage electrical circuit is according like proposed in which makes the assumption that the current density is uniform in the section of the conductor. Note $\mathbf{j}_{0}^{s}(x, y, z)$ the space function of the corresponding current density which is obtained by the electrokinetic resolution of the problem with the total current is $1 A$ [6]. In the case where the total current 
TABLE I

BEM FORMULATIONS FOR EC PROBLEM

\begin{tabular}{ccc}
\hline \hline Formulation & Factors & Variables \\
\hline \multirow{2}{*}{ EC1 } & $\alpha_{1}=1, \alpha_{0}=\beta_{1}=0, \beta_{0}=1$ & $\mathbf{J}_{s}, \mathbf{M}_{s}$, \\
& $a_{1}=a_{0}=0, b_{1}=0, b_{0}=1$ & $\mathbf{H}_{0 n}$ \\
\hline \multirow{2}{*}{ EC2 } & $\alpha_{1}=0, \alpha_{0}=\beta_{1}=1, \beta_{0}=0$ & $\mathbf{J}_{s}, \mathbf{M}_{s}$, \\
& $a_{1}=0, a_{0}=b_{1}=1, b_{0}=0$ & $\mathbf{H}_{0 n}, \mathbf{E}_{0 n}$ \\
\hline \multirow{2}{*}{ EC3 } & $\alpha_{1}=\alpha_{0}=\beta_{1}=\beta_{0}=1$ & $\mathbf{J}_{s}, \mathbf{M}_{s}$, \\
& $a_{1}=0, a_{0}=1, b_{1}=b_{0}=0$ & $\mathbf{E}_{0 n}$ \\
\hline \multirow{2}{*}{ EC4 } & $\alpha_{1}=\alpha_{0}=\beta_{1}=\beta_{0}=1$ & $\mathbf{J}_{s}, \mathbf{M}_{s}$ \\
& $a_{1}=0, a_{0}=1, b_{1}=b_{0}=0$ & $\mathbf{J}_{s}, \mathbf{M}_{s}$, \\
\multirow{2}{*}{ EC5 } & $\alpha_{1}=-1, \alpha_{0}=1, \beta_{1}=-\mu_{1}, \beta_{0}=\mu_{0}$ & $\mathbf{E}_{0 n}$ \\
\hline \multirow{2}{*}{ EC6 } & $a_{1}=-1, a_{0}=1, b_{1}=b_{0}=0$ & $\mathbf{J}_{s}, \mathbf{M}_{s}$, \\
& $\alpha_{1}=-1, \alpha_{0}=1, \beta_{1}=-1, \beta_{0}=1$ & $\mathbf{E}_{0 n}$ \\
\hline \hline
\end{tabular}

is equal to $I$ with a voltage imposed $U$ on the conductor $U=V_{a}-V_{b}$ with $V_{a}, V_{b}$ respectively the uniform potential on borders $\Gamma_{a}, \Gamma_{b}$, thanks to Stock's theorem the following relationship can be written:

$$
\int_{\Omega^{s}} \mathbf{j}_{0}^{s} \cdot \nabla V d \Omega=V_{a} \int_{\Gamma_{a}} \mathbf{j}_{0}^{s} \cdot \mathbf{n} d \Gamma+V_{b} \int_{\Gamma_{b}} \mathbf{j}_{0}^{s} \cdot \mathbf{n} d \Gamma=-U
$$

The electric field $\mathbf{E}$ in the conductor is written as:

$$
\mathbf{E}=-j \omega \mathbf{A}-\nabla V
$$

In combination with the above equation and (14), we get:

$$
U=\int_{\Omega^{s}} \mathbf{j}_{0}^{s} \cdot \mathbf{E} d \Omega+j \omega \int_{\Omega^{s}} \mathbf{j}_{0}^{s} \cdot \mathbf{A} d \Omega
$$

The term of $\mathbf{j}_{0}^{s} \cdot \mathbf{E}$ can be written as:

$$
\int_{\Omega_{c}} \mathbf{j}_{0}^{s} \cdot \mathbf{E} d \Omega=-\int_{\Omega_{c}} \mathbf{j}^{s} \cdot \nabla V_{0} d \Omega=U_{0} I=R I
$$

where $R$ is static resistance and $R=\rho \int_{\Omega_{c}}\left(\mathbf{j}_{0}^{s}\right)^{2} d \Omega$.

Equations (16) and (17) lead to the following electrical circuit equation :

$$
U=\int_{\Omega^{s}} \rho I\left(\mathbf{j}_{0}^{s}\right)^{2} d \Omega+j \omega \int_{\Omega^{s}} \mathbf{j}_{0}^{s} \cdot \mathbf{A} d \Omega
$$

The total magnetic potential $\mathbf{A}$ in $(18)$ is created by both current flowing in the electric circuit and by eddy currents.

\section{B. Coupling model}

The circuit coupling is implemented with the assumption that the excitation circuits are wound conductors where the wire size is small enough to not need to take into account the skin effect in each strand. In the context of circuit coupling, the conducting region $\Omega$ is excited by a set of $n$ electrical circuits in which the $k^{t h}$ electrical circuit $\Omega_{k}^{s}$ is connected to voltage sources $U_{k}$. The governing equation is (18). Let us consider (2). By setting the coefficient $h$ to 1 , we get the electrical total field outside the considered conductor $\Omega$ :

$$
\begin{aligned}
\mathbf{E}= & -j \omega \mu_{0} \sum_{i=1}^{n} I_{i} \int_{\Omega_{i}^{s}} \mathbf{j}_{0 i}^{s} G_{0} d \Omega-j \omega \mu_{0} \int_{\Gamma} \mathbf{J}_{s} G_{0} d \Gamma \\
& -\int_{\Gamma} \mathbf{M}_{s} \times \nabla G_{0} d \Gamma+\int_{\Gamma} \mathbf{E}_{n 0} \nabla G_{0} d \Gamma
\end{aligned}
$$

Thanks to the relation $\mathbf{E}=-j \omega \mathbf{A}$, the equation governing the $k^{\text {th }}$ electric circuit becomes:

$$
\begin{aligned}
& U_{k}=I_{k} \underbrace{\left(\rho \int_{\Omega_{k}^{s}}\left(\mathbf{j}_{0 k}^{s}\right)^{2} d \Omega+j \omega \mu_{0} \int_{\Omega_{k}^{s}} \mathbf{j}_{0 k}^{s} \cdot \int_{\Omega_{k}^{s}} \mathbf{j}_{0 k}^{s} G_{0} d \Omega d \Omega\right)}_{\text {Term I }} \\
& \underbrace{+j \omega \mu_{0} \int_{\Omega_{k}^{s}} \mathbf{j}_{0 k}^{s} \sum_{i \neq k}^{n} I_{i} \int_{\Omega_{i}^{s}} \mathbf{j}_{0 i}^{s} G_{0} d \Omega d \Omega}_{\text {Term } 2} \\
& -\underbrace{\int_{\Omega_{k}^{s}} \mathbf{j}_{0 k}^{s}\left(-j \omega \mu_{0} \int_{\Gamma} \mathbf{J}_{s} G_{0} d \Gamma-\int_{\Gamma} \mathbf{M}_{s} \times \nabla G_{0} d \Gamma+\int_{\Gamma} \mathbf{E}_{n 0} \nabla G_{0} d \Gamma\right) d \Omega}_{\text {Term } 3}
\end{aligned}
$$

Term 1 presents the proper impedance of the $k^{t h}$ electrical circuit. Term 3 and Term 2 express the reaction of eddy currents and the influences of others electrical circuits on the $k^{\text {th }}$ electrical circuit respectively.

\section{Resolution}

Both eddy current BEM formulations and their coupling with electric circuits are solved simultaneously. According to (5), the electric and magnetic source fields in BEM formulations can be represented by integral depending on $I_{k}$ which are additional unknowns. The final matrix system to solve is presented in Fig. 2.

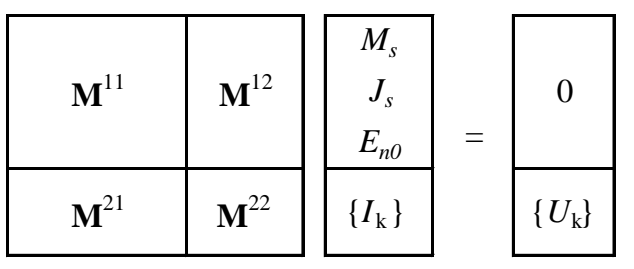

Fig. 2. Matrix system for solving the coupling problem.

In the system, $M_{s\left(n_{M} \times 1\right)}, J_{s\left(n_{J} \times 1\right)}, E_{n 0\left(n_{E} \times 1\right)}$ are vectors of the degrees of freedom corresponding to $\mathbf{M}_{s}, \mathbf{J}_{s}$ and $\mathbf{E}_{n 0}$. The matrix $\mathbf{M}^{11}$ is constructed as the one used to solve the single eddy current problem by BEM formulation. The $\mathbf{M}^{11}$ matrix is built like the one used to solve the eddy current problem by BEM formulation. Let's establish the matrices $\mathbf{M}^{12}, \mathbf{M}^{21}$ and $\mathbf{M}^{22}$. The current density $\mathbf{j}_{0 k}^{s}$ is interpolated also by the facet shape function:

$$
\mathbf{j}_{0 k}^{s}=\sum_{i=1}^{n_{k}} \mathbf{w}_{k i} j_{k i}
$$

where $j_{k i}$ can be determinded thanks to the pre-computation of the electrokinetic current density $\mathbf{j}_{0 k}$ discussed in the section III.B.

Suppose that the $\mathbf{M}^{21}$ matrix is composed of three parts $\mathbf{N}^{1}$, $\mathbf{N}^{2}, \mathbf{N}^{3}$ corresponding to three sets of unknown $M_{s}, J_{s}, E_{n 0}$ respectively. The $k^{\text {th }}$ row of these matrices corresponds to the Term 3 of equation of the $k^{t h}$ electrical circuit. 


$$
\begin{aligned}
& \mathbf{N}_{k j}^{1}=j \omega \mu_{0} \sum_{i=1}^{n_{k}}\left(j_{k i} \int_{\Omega_{k}^{s}} \mathbf{w}_{k i} \int_{\Gamma} \mathbf{w}_{j} G_{0} d \Gamma d \Omega\right) \\
& \mathbf{N}_{k j}^{2}=\sum_{i=1}^{n_{k}}\left(j_{k i} \int_{\Omega_{k}^{s}} \mathbf{w}_{k i} \int_{\Gamma} \mathbf{w}_{j} \times \nabla G_{0} d \Gamma d \Omega\right) \\
& \mathbf{N}_{k j}^{3}=-\sum_{i=1}^{n_{k}}\left(j_{k i} \int_{\Omega_{k}^{s}} \mathbf{w}_{k i} \int_{\Gamma} \alpha_{j} \nabla G_{0} d \Gamma d \Omega\right)
\end{aligned}
$$

The matrix $\mathbf{M}^{22}$ is constructed according to Term 1 and Term 2. Its $k^{\text {th }}$ row takes the following form:

$$
\begin{aligned}
& \mathbf{M}_{k k}^{22}=\rho \sum_{i=1}^{n_{k}} j_{k i} \sum_{j=1}^{n_{k}} j_{k j} \int_{\Omega_{k}^{s}} \mathbf{w}_{k i} \mathbf{w}_{k j} d \Omega \\
& \mathbf{M}_{k t}^{22}=j \omega \mu_{0} \sum_{i=1}^{n_{k}} j_{k i} \sum_{j=1}^{n_{t}} j_{t j} \int_{\Omega_{k}^{s}} \mathbf{w}_{k i} \int_{\Omega_{t}^{s}} \mathbf{w}_{t j} G_{0} d \Omega d \Omega
\end{aligned}
$$

$\mathbf{M}^{12}$ is established in the same way as $\mathbf{M}^{22}$, follows the source formula (5).

\section{Validation}

The circuit coupling has been tested with EC6 formulation. A conductive square section ring is taken into account. The system is excited by an external circuit, which is concentric and coaxial with the ring. The geometric and property descriptions are shown in Tab. II and Fig. 3 In BEM, the geometry is meshed with about 2464 quadrangle elements. The reference is calculated by the FEM method with a 2D axisymmetric model and a circuit coupling in which a mesh of 43500 triangles is used. The total currents flowing in the electrical circuit are compared in Tab. IIII The magnetic flux density is calculated on line segment $A(28.5,0,5)-B(28.5,0,30)$ at frequency $f=10 \mathrm{~Hz}$ and shown in Fig. 4 The component $\mathbf{B}_{y}=0$ is not displayed in the figure. The eddy current distribution in this case is shown in Fig 3 .

TABLE II

PROBLEM DESCRIPTIONS

\begin{tabular}{lclc}
\hline \hline Ring with square section & Electrical circuit & \\
\hline Conductivity & $3.526 \mathrm{E} 7(\mathrm{~S} / \mathrm{m})$ & Imposed voltage & $10(\mathrm{~V})$ \\
Relative permeability & 1 & Height/width & $5(\mathrm{~mm})$ \\
Height & $60(\mathrm{~mm})$ & Number of turns & 100 \\
External radius & $25(\mathrm{~mm})$ & External radius & $31(\mathrm{~mm})$ \\
Internal radius & $10(\mathrm{~mm})$ & Internal radius & $26(\mathrm{~mm})$ \\
\hline \hline
\end{tabular}

TABLE III

CURRENT IN THE EXTERNAL ELECTRICAL CIRCUIT

\begin{tabular}{lcccc}
\hline \hline \multicolumn{2}{l}{ Frequency (Hz) } & 10 & 1000 & 10000 \\
\hline \multirow{2}{*}{ BEM Current (A) } & $-2.4586+0.0353 \mathrm{j}$ & $-1.4166+1.0339 \mathrm{j}$ & $-0.0726+0.3331 \mathrm{j}$ \\
& Mod(I) (A) & 2.4589 & 1.7536 & 0.3409 \\
Ref. & Current (A) & $-2.4585+0.0353 \mathrm{j}$ & $-1.4257+1.0260 \mathrm{j}$ & $-0.0765+0.3385 \mathrm{j}$ \\
\multicolumn{2}{l}{ Mod(I) (A) } & 2.4588 & 1.7565 & 0.3470 \\
\multicolumn{2}{l}{ Dif. of $\operatorname{Mod}(\mathrm{I})(\%)$} & 0.0028 & 0.1570 & 1.7603 \\
\hline \hline
\end{tabular}

The BEM circuit coupling leads to results very close to those calculated by FEM method with a axisymetric model. Differences can be explained by the difference of the mesh
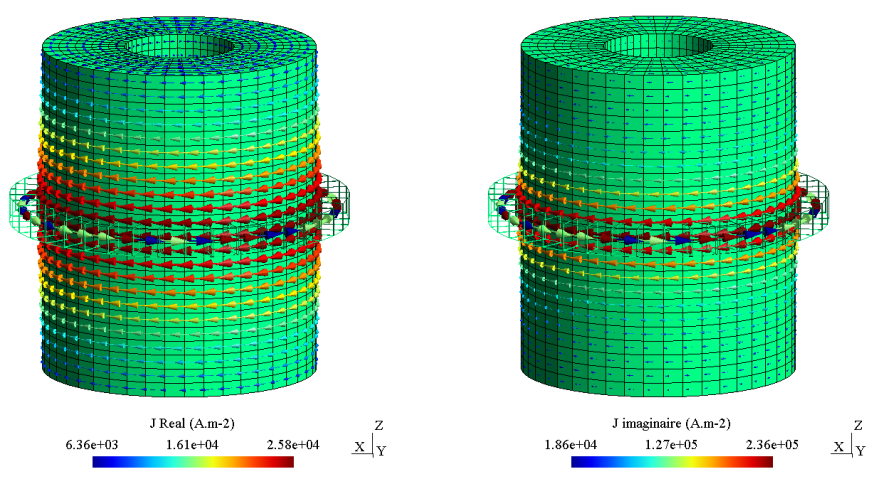

Fig. 3. Eddy current distribution $\mathbf{J}\left(A \cdot m^{-} 2\right)$ on the surface of the ring at $f=10 H z$

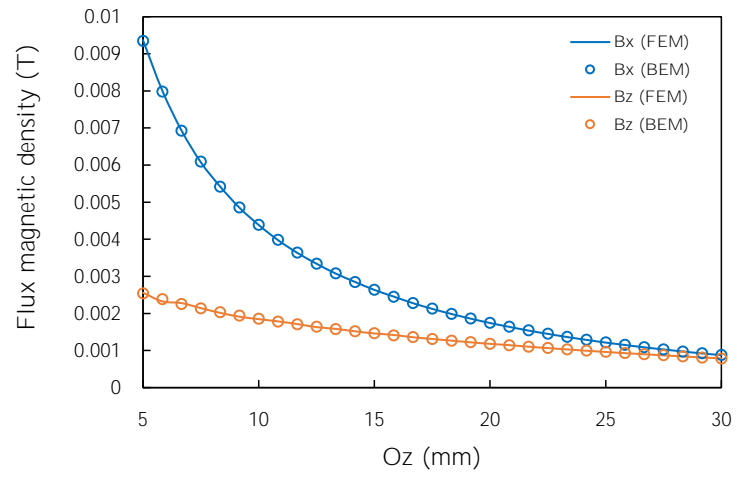

Fig. 4. Flux magnetic density calculated on line segment $\mathrm{AB}$ at $f=10 \mathrm{~Hz}$

size and by the order of the shape functions ( first order for BEM functions and second order for FEM) but results remains very goods.

\section{CONCLUSION}

In this article, different formulations for the eddy current problem have been recalled and compared at low frequency. These formulations generally work well with low relative permeability. A coupling model that simultaneously solves the eddy current and electrical circuit equations has also been proposed.

\section{REFERENCES}

[1] Vigneron A. Formulations by surface integral equations for numerical simulation of non-destructive testing by eddy currents. https://hal.archives-ouvertes.fr/tel-01114368.

[2] A. Vigneron, E. Demaldent and M. Bonnet, "Surface Integral Equations for Electromagnetic Testing: The Low-Frequency and High-Contrast Case," in IEEE Transactions on Magnetics, vol. 50, no. 2, pp. 117-120, Feb. 2014, Art no. 7002704. doi: 10.1109/TMAG.2013.2283297

[3] W. M. Rucker, R. Hoschek and K. R. Richter, "Various BEM formulations for calculating eddy currents in terms of field variables," in IEEE Transactions on Magnetics, vol. 31, no. 3, pp. 1336-1341, May 1995. doi: 10.1109/20.376274

[4] Dalian Zheng, "Three-dimensional eddy current analysis by the boundary element method," in IEEE Transactions on Magnetics, vol. 33, no. 2, pp. 1354-1357, March 1997. doi: 10.1109/20.582507

[5] Hiptmair, Ralf. (2001). Boundary Element Methods for Eddy Current Computation. Lecture Notes in Applied and Computational Mechanics. 2007. 10.1007/978-3-540-47533-0_9.

[6] G. Meunier, H. T. Luong and Y. Marechal, "Computation of coupled problem of 3D eddy current and electrical circuit by using T/sub $0 /-\mathrm{T}-$ /spl phi/ formulation," in IEEE Transactions on Magnetics, vol. 34, no. 5, pp. 3074-3077, Sept. 1998. doi: 10.1109/20.717719

[7] Muller, Cl. (1969). Foundations of the Mathematical Theory of Electromagnetic Waves. 10.1007/978-3-662-11773-6. 